\title{
Macromolecule Extravasation-Xenograft Size Matters: A Systematic Study Using Probe-Based Confocal Laser Endomicroscopy (pCLE)
}

\author{
Antje Dietrich, ${ }^{1}$ James Stewart, ${ }^{2}$ Melanie Huether, ${ }^{1}$ Mario Helm, ${ }^{3}$ Christina Schuetze, ${ }^{4}$ \\ Hans-Joachim Schnittler, ${ }^{5}$ David A. Jaffray, ${ }^{2,6}$ Leoni A. Kunz-Schughart ${ }^{1}$ \\ ${ }^{1}$ Tumor Pathophysiology, OncoRay-National Center for Radiation Research in Oncology, Medical Faculty Carl Gustav Carus, \\ Fetscherstraße 74 P.O. Box 4101307 TU Dresden, Germany \\ ${ }^{2}$ Institute of Biomaterials and Biomedical Engineering, University of Toronto, Toronto, ON, Canada \\ ${ }^{3}$ Medical Radiation Physics, OncoRay-National Center for Radiation Research in Oncology, Medical Faculty Carl Gustav Carus, TU \\ Dresden, Germany \\ ${ }^{4}$ Experimental Radiotherapy and Radiobiology of Tumors, OncoRay-National Center for Radiation Research in Oncology, Medical \\ Faculty Carl Gustav Carus, TU Dresden, Germany \\ ${ }^{5}$ Department of Anatomy and Cell Biology, Institute of Anatomy, University of Muenster, Muenster, Germany \\ ${ }^{6}$ Radiation Medicine Program, Princess Margaret Hospital/Ontario Cancer Institute, University Health Network, Toronto, ON, Canada
}

\begin{abstract}
Purpose: Profound changes of the vasculature in tumors critically impact drug delivery and therapy response. We aimed at developing a procedure to monitor morphological and functional parameters of the vasculature in subcutaneous xenograft models commonly applied for therapy testing by using probe-based confocal laser endomicroscopy.

Procedures: By monitoring various normal and diseased tissues, we established an experimental and analytical set-up to systematically analyze tracer extravasation from the microvasculature. Application of the approach in two xenograft models (HCT-116 and SW620) was realized consecutively throughout tumor growth.

Results: The incidence of dilated vessels increased with xenograft size in both models while macromolecule extravasation and tracer accumulation in the tumor tissue, respectively, was significantly reduced throughout growth. The development of dilated/ultradilated vessels correlated with tracer extravasation only in the HCT-116 but not the SW620 model. The underlying mechanisms are still ambiguous and discussed.

Conclusions: Our findings clearly indicate that both xenograft type and size matter for drug delivery and therapy testing.
\end{abstract}

Key words: Macromolecule extravasation, Probe-based confocal fluorescence microscopy, In vivo imaging, Tumor vasculature, Xenograft growth

Electronic supplementary material. The online version of this article (doi:10.1007/s11307-013-0641-z) contains supplementary material, which is available to authorized users.

Correspondence to: Leoni A. Kunz-Schughart; e-mail: leoni.kunzschughart@oncoray.de 
Abbreviations: $\mathrm{AVH}$, Acute vascular hyperpermeability; BVP, Basal vascular permeability; $\mathrm{CVH}$, Chronic vascular hyperpermeability; pCLE, Probe-based confocal laser endomicroscopy; FITC, Fluorescein isothiocyanate; IFP, Interstitial fluid pressure

\section{Introduction}

$\mathrm{P}$ athological conditions like inflammation, wound healing, or cancer are characterized by the formation of new vessels initiated by the production of proangiogenic cytokines [1]. After tissue injury, permeabilizing factors which are primarily stored in mast cells (e.g., histamine and VEGF) are released and trigger a process called acute vascular hyperpermeability (AVH) - a rapid influx of an exudate consisting mainly of plasma proteins which is selflimited and attenuates within $30 \mathrm{~min}$ [2]. Angiogenesis is terminated after healing resulting in healthy vasculature [3]. In contrast, tumors are often compared to chronic inflammation and wounds that do not heal [4, 5]. Here, exposure to angiogenic factors is not limited which causes persistent angiogenesis with vessels that show profound morphological and functional anomalies including chronic vascular hyperpermeability $(\mathrm{CVH})[2,6]$. It was reported that macromolecules larger than $40 \mathrm{kDa}$ extravasate in solid tumors in contrast to normal tissues [7]. This enhanced permeability and retention effect (EPR effect) is the basis for the development of macromolecular tumor-targeting drugs as they show prolonged plasma half-life and higher tumor selectivity compared to conventional chemotherapeutics $[7,8]$.

It is of utmost interest to gain deeper insight in the pathophysiology of tumor vasculature and to develop tools to better image and understand changes in vascular function, particularly during therapy testing. The application of conventional microscopy during an appointed time course requires substantial numbers of animals [9], while methods like MRI, CT, or optical fluorescence imaging do not reach spatial microscopic resolutions [10, 11]. Today, diverse window chamber preparations are applied to analyze vessels and vascular permeability via intravital microscopy [12-14]. A disadvantage of these approaches is their high invasiveness and the requirement of demanding surgical procedures [15] as well as the limited tumor size which makes them inappropriate for therapy testing. Probe-based confocal laser endomicroscopy (pCLE; Cellvizio $^{\circledR}$ technology) offers the possibility for real-time imaging of microvessels with minimal invasiveness and at microscopic resolution [16-18]. Thereby, standard models as used for therapy testing (e.g., subcutaneous xenografts with diameters $>0.5 \mathrm{~cm}$ ) are becoming accessible for in vivo microscopy.
Controversial results of studies on therapy testing in animal models could be caused by methodical inconsistencies [19]. For instance, there is no consensus about the sizes of xenografts that enter treatment studies, although this may critically impact the outcome because tumor characteristics such as local microenvironment change throughout growth. We were particularly interested in variations of vascular morphology and macromolecule extravasation during xenograft growth as those parameters can affect the delivery and accumulation of putative anti-cancer drugs. We applied pCLE to monitor vasculature in healthy and diseased tissue in mouse models and developed a protocol to visualize and estimate the extravasation and accumulation of a fluorescein isothiocyanate (FITC)-labeled dextran tracer with the aim to distinguish acute and chronic hyperpermeability. Based on our experimental set-up, we for the first time systematically studied morphology and tracer permeation in two independent xenograft models longitudinally throughout tumor growth.

\section{Methods}

C57BL/6 mice (12-16 weeks old) were used in the wound healing experiments as described earlier in Peters et al. [20]. Experiments on xenograft tumors were performed using 8-14-week-old female NMRI-nu/nu mice for subcutaneous injection of HCT-116 and SW620 colorectal cancer cells (ATCC). The animal facility and all experiments were approved in accordance to institutional guidelines and German animal welfare regulations.

Probe-based confocal laser endomicroscopy (pCLE, Leica FCM1000; Leica Microsystems/Mauna Kea Technologies) was applied on anesthetized mice using intravenously injected FITC-dextrans (tail vein, 150 or $500 \mathrm{kDa}, 3.5 \mathrm{mg}$ per mouse; Sigma-Aldrich) as tracers. The protocol to estimate tracer extravasation is detailed in Fig. 1a and was used at three tumor sizes longitudinally during xenograft growth. A leak index, which relates the average extravascular fluorescence intensity $I_{\mathrm{En}}$ to the intravascular signal $I_{\mathrm{Vn}}$, was calculated by analyzing at least seven different fields of view (FOV) per tumor and time point including five consecutive frames per FOV. Vessels were segmented in the first movie sequences after tracer injection by iteratively segmenting each frame of the movie (Fig. 1b) with custom-made software written in MATLAB (The MathWorks). Due to lack of suitable FOVs and loss of mice during xenograft growth, it was not possible to include all tumors that entered the experiment in the analysis of each size range. However, the results of the longitudinal studies are obtained from the same cohort of xenograft tumors $(n=12$ for HCT-116 and $n=23$ for SW620). 
a

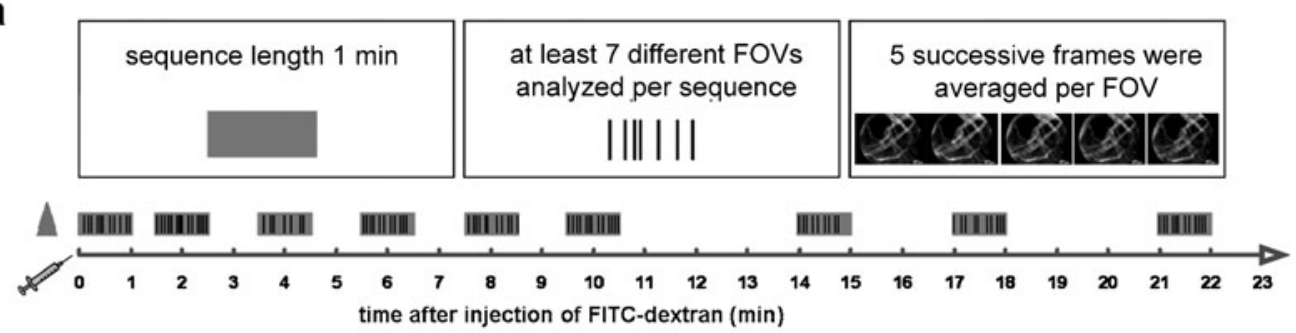

b

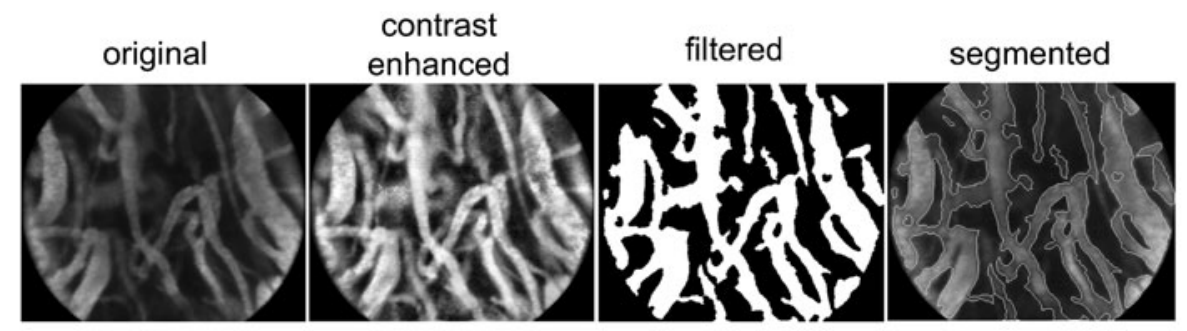

c

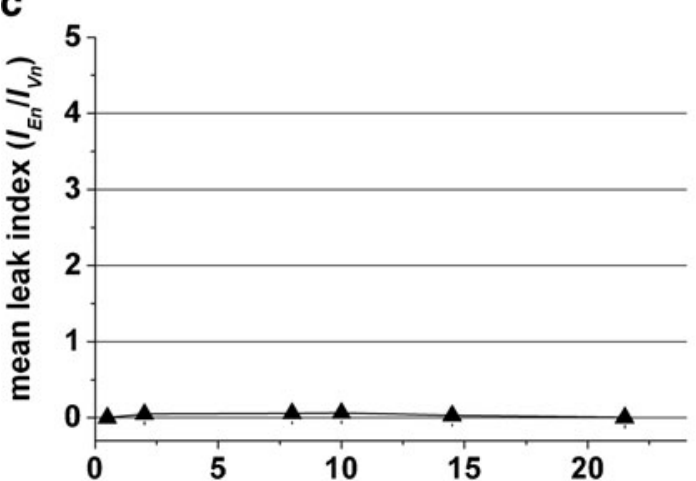

time after injection of FITC-dextran (min) d $\quad \Delta$ tongues of control mice

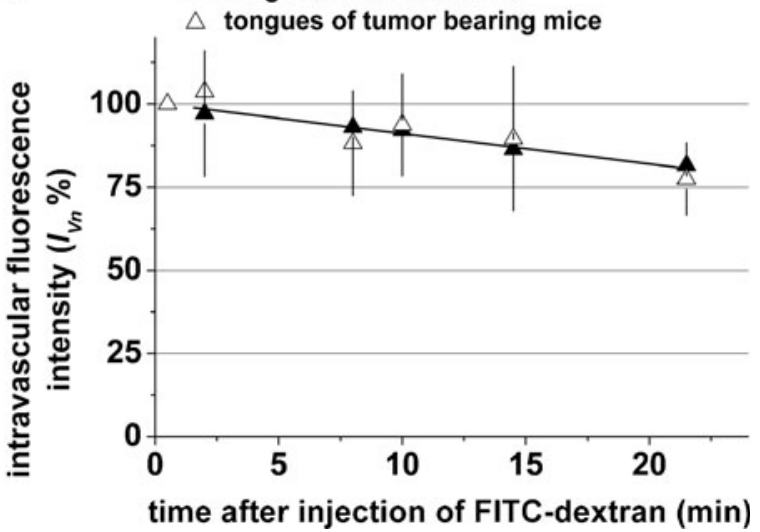

Fig. 1. Prerequisites to analyze macromolecule extravasation via pCLE. a Schematic illustration of the protocol to determine tracer extravasation. To expose xenografts or muscles, a skin incision $(\Lambda)$ was required and directly followed by i.v. injection of $150 \mathrm{kDa}$ FITC-dextran ( ) into the tail vein. One-minute sequences ( ) were recorded at defined time points after injection. Areas which were recorded on five consecutive images were examined offline and the data was averaged for each FOV. At least seven different FOVs (|) were imaged, thus, a minimum of 35 frames ( 5 frames $\times 7$ FOVs) were analyzed per time point and animal. b Illustration of vessel segmentation for an individual frame. c Mean leak indices $\left(I_{\mathrm{En}} / /_{\mathrm{Vn}} \pm \mathrm{SD}\right)$ of tongues of $n=8 \mathrm{NMRI}$ $\mathrm{nu} / \mathrm{nu}$ mice under normal conditions were estimated, see also Suppl. Fig. S5. d Intravascular signal $\left(I_{V n}, \pm S D\right)$ in tongues decreases slightly during monitoring. This clearance is independent from presence of a tumor $(n=4)$ and can be corrected for [linear fit $(n=8), y=-0.9352 x+100 ; r=0.992]$.

Detailed information on animal models, the pCLE system, and the developed protocols including practical procedure, data analysis, and image segmentation is given in the Electronic Supplementary Material.

\section{Results}

\section{Monitoring Healthy and Diseased Vessel Networks In Vivo via $p C L E$}

By injection of FITC-dextran $(500 \mathrm{kDa})$, we monitored the vascular morphology in different tissues in NMRI-nu/nu mice via pCLE (Suppl. Fig. S1a). As revealed in tongue tissue, the tracer distributed in the vasculature within a few seconds (Video 1) and circulated in the blood. The movement of unlabeled blood cells permits to follow the blood flow in the recorded movie sequences (Videos $2 \mathrm{a}$ and $\mathrm{b}$ ).

A wound healing model in C57BL/6 mice was used to test the applicability of pCLE for visualization of vascular recovery in vivo. Clear changes in the profile of the vasculature in wound margins were observed (Suppl. Fig. S1b). At day 3 after wounding, vessels were dilated and an increase in extravascular signal indicated an enhanced vascular permeability. Seven days after wound initiation, vessel morphology had somewhat normalized, but hyperpermeability had not yet fully disappeared as was the case at day 12 after wounding. 

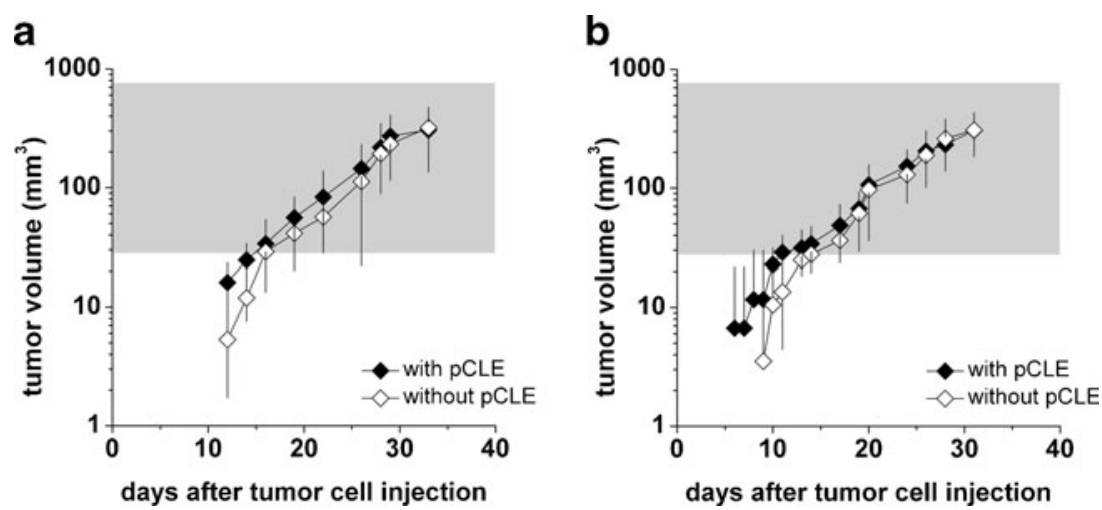

Fig. 2. The pCLE imaging protocol does not affect HCT-116 tumor growth kinetics. Comparison of tumor volumes of HCT-116 xenografts in NMRI-nu/nu mice that were either imaged twice with pCLE during tumor growth or untouched $(n=5$ tumors for each group); $500 \mathrm{kDa}$ a and $150 \mathrm{kDa}$ b FITC-dextran was used as respective tracer molecule during acquisition. The range of tumor volume where the measurements were applied is shaded in gray. The tumor volumes of the respective groups did not significantly differ at any time point as was determined using two-sided Student's $t$ test.

We also applied pCLE to subcutaneous xenograft tumor models in NMRI-nu/nu mice. In general, a strikingly high intertumoral heterogeneity within each model was observed (Suppl. Fig. S1c). Intratumoral heterogeneity of vascular networks was also high in some but not all xenografts (Suppl. Fig. S1d), stressing the necessity to monitor numerous FOVs for subsequent quantitative analyses. As expected, tumor vessels showed a chaotic morphology, were of highly variable size (length and diameter), and exhibited tortuous courses without hierarchical order. Vessel constrictions or dead ends were seen in some tumors (Suppl. Fig. S3 and Video 3). Dilated vessels with diameters $>50 \mu \mathrm{m}$ were frequent and possessed serpentine or linear nature with different and altering rates of blood flow (Videos $4 a$ and $b$ ).

We compared the growth kinetics of xenografts that were monitored twice throughout growth with pCLE with respective untouched controls to exclude an impact of anesthesia, skin incisions, tracer injections, and laser illuminations on tumor growth. Fig. 2 documents that the implemented protocol did not affect HCT-116 xenograft growth. We also found that FITC-dextrans were no longer detectable in the majority of tumors and normal tissues (>95\%) 3 days after injection. Vessel monitoring with the technology can thus be realized repeatedly over a certain time course.

\section{Monitoring Extravasation in Normal Tissue}

The $500 \mathrm{kDa}$ FITC-dextran used in previous experiments to visualize the vascular morphology infrequently extravasated, e.g., after wounding, but seemed suboptimal for studying tracer leakage. In contrast, $150 \mathrm{kDa}$ FITC-dextran (3.5 mg per mouse) was found to fulfill all requirements for both vessel detection and tracking extravasation. Initially, healthy tongue tissue under normal conditions without external stimulus was analyzed for extravasation of i.v. injected $150 \mathrm{kDa}$ FITC-dextran (Fig. 1c). The tracer did not extravasate under those conditions as reflected by an unchanged leak index (Fig. 1c and Suppl. Fig. S5). This result was expected because the basal vascular permeability (BVP) of normal microvessels is characterized by diffusion of a plasma filtrate with only little amount of larger molecules. Because extravascular fluorescence intensity and image contrast remained stable, it was possible to apply the vessel segmentation algorithm at all time points and to monitor changes in vascular fluorescence intensity $I_{\mathrm{V}}$ during the acquisition period. As expected, $I_{\mathrm{V}}$ showed a slight decrease due to tracer plasma clearance. This was independent from the presence of a subcutaneous tumor (Fig. 1d). The comparison of linear and exponential regressions revealed overlapping functions for the time interval of interest of $\sim 25 \mathrm{~min}$ (linear fit $y=-0.9352 x+100, r=0.992$ and exponential fit $y=100 e-0.0102 x, r=0.976$, respectively). According to the correlation coefficients, the linear equation was sufficient to correct $I_{\mathrm{Vn}}$ in all further reported measurements.

To verify if a strong increase in vascular permeability caused by a particular event (AVH) can be visualized via pCLE, tongues were pinched with the forceps directly prior to the injection of $150 \mathrm{kDa}$ FITC-dextran, and the probe was then moved over the resulting lesion. The leak index shown in Fig. 3a reflects the rapid influx of FITCdextran into the surrounding tissue. This reaction was restricted to the pinched regions and was already seen during the first time point in some FOVs. Therefore, it was necessary to collect the images for calculation of vascular fraction and $I_{\mathrm{V}}$ from regions that were not affected by the pinching. Another group of animals was included whose tongues were pinched as described, but tracer injection and vessel monitoring started $1 \mathrm{~h}$ later. At this time point, no FITC-dextran extravasation was detectable indicating the termination of the acute reaction (Fig. 3a). The transient nature of this type of hyperpermeability and its dependence on a stimulus substantiates that $\mathrm{AVH}$ was induced. 
a

a $\quad \mathrm{AVH}$ in tongues
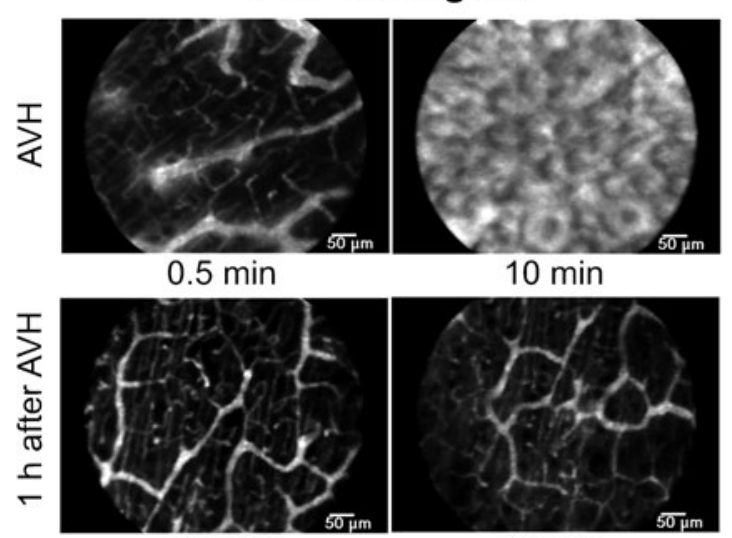

$0.5 \mathrm{~min}$

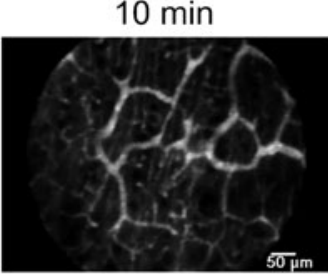

10 min

b
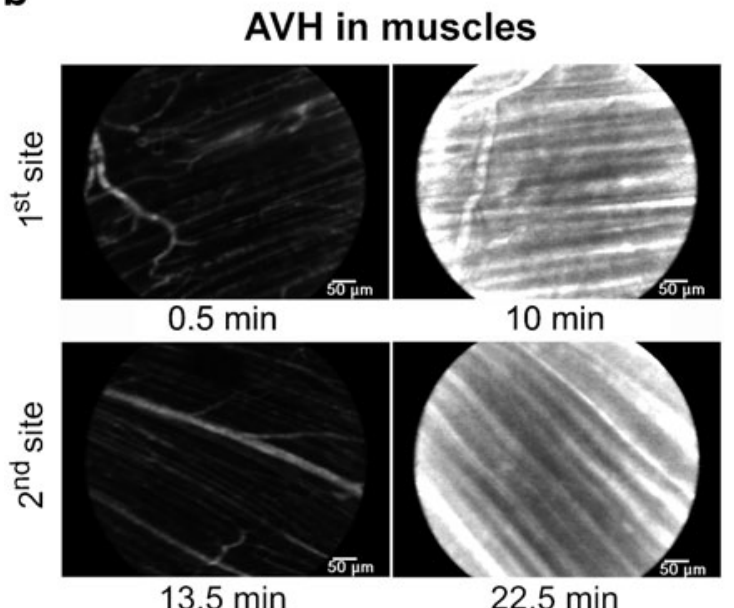

$13.5 \mathrm{~min}$

$22.5 \mathrm{~min}$

C

$\mathrm{CVH}$ in xenograft tumors

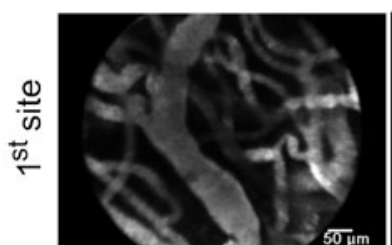

$0.5 \mathrm{~min}$

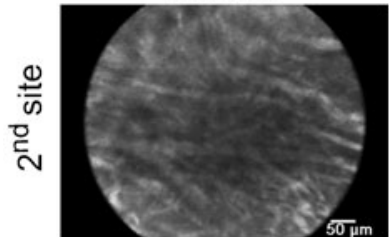

$13.5 \mathrm{~min}$

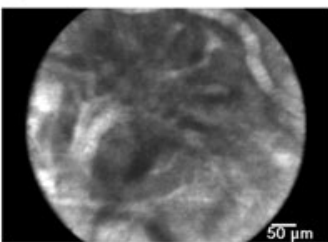

$10 \mathrm{~min}$

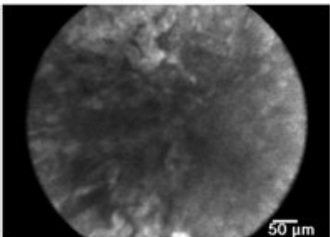

$22.5 \mathrm{~min}$
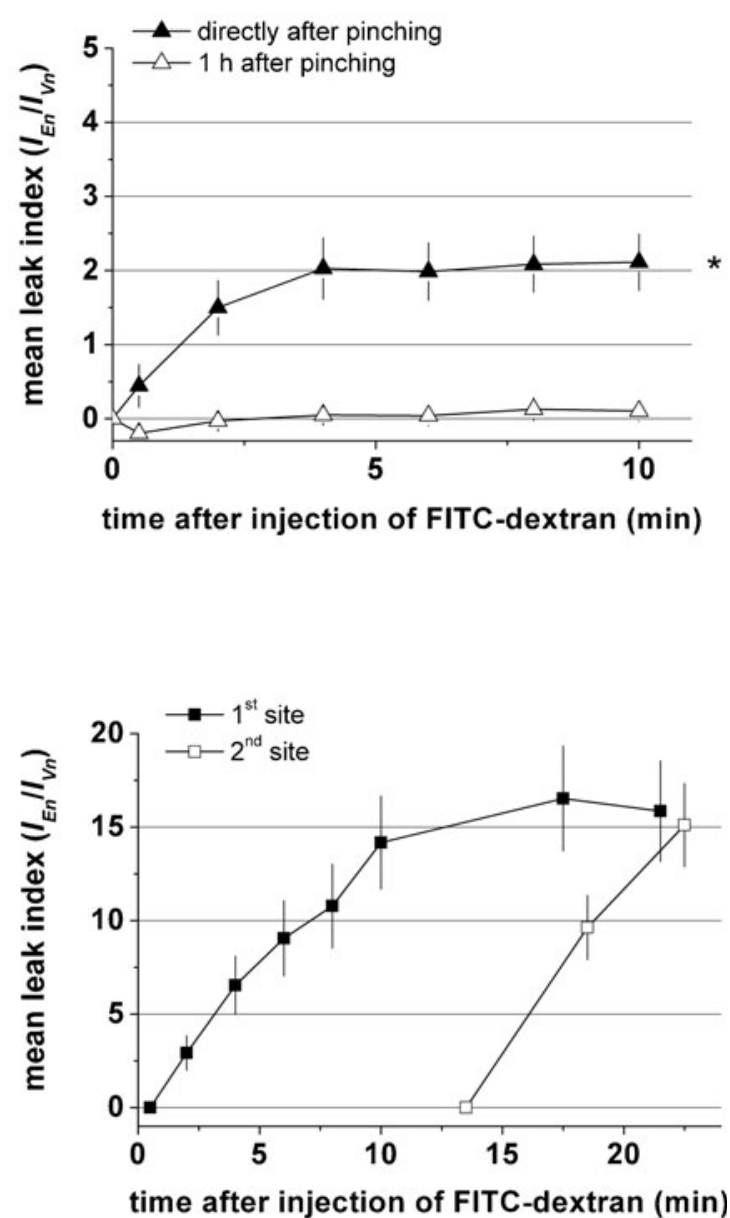

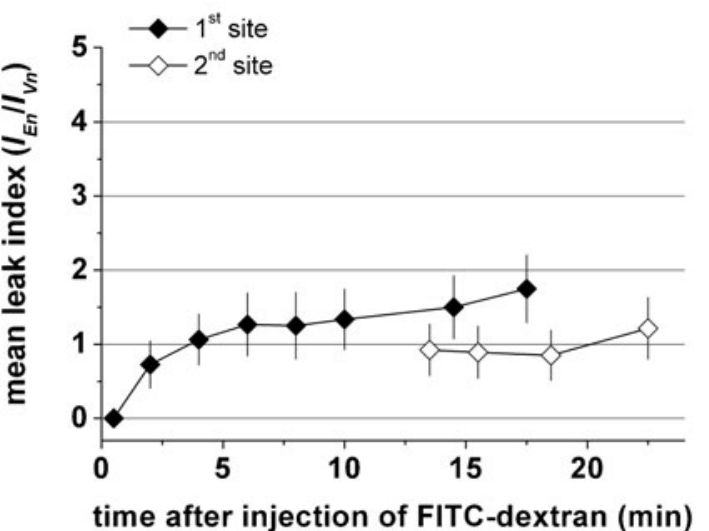

Fig. 3. The proposed experimental protocol allows monitoring of AVH in normal tissues and CVH in xenografts. Representative images of vasculature of tongues (a, $n=6)$, femur muscles ( $\mathbf{b}, n=7)$, and subcutaneous HCT-116 xenografts (c, $n=6)$ of NMRI$\mathrm{nu} / \mathrm{nu}$ mice at different time points after $150 \mathrm{kDa}$ FITC-dextran injection and the corresponding mean leak indices $\left(I_{\mathrm{En}} / /_{\mathrm{Vn}}\right)$. Error bars show the SD from $n$ measurements. a Tongues were pinched and imaged either directly or one hour delayed. $\mathbf{b}, \mathbf{c}$ Tracer was injected directly after skin incision at the first site whereas the second site was injured and imaged 12 min later. ${ }^{*} p<0.05$, two-sided Student's $t$ test. 
To monitor extravasation of the tracer at the muscle site, a small skin incision was necessary for direct access to the tissue surface. As documented in Fig. 3b, rapid and massive extravasation of the tracer occurred shortly after injection, which evidently indicates an AVH reaction most reasonably caused by the wounding. The second femur muscle of the mice was included in the monitoring regime for approval. The skin of the second leg was incised 12 min after FITCdextran injection and the measurement was thus delayed. As expected, tracer extravasation started only after wounding at the specific site and was completely independent from the time point of FITC-dextran injection (Fig. 3b). The inducible characteristic of this reaction and the healthy morphology of the vasculature thus reveal the presence of AVH.

\section{Chronic Vascular Hyperpermeability Detected in Xenograft Tumors}

We then utilized the protocol to monitor extravasation of $150 \mathrm{kDa}$ FITC-dextran in our subcutaneous HCT-116 xenograft model. The images and leak index values documented in Fig. 3c (first site) indicate permeation of the macromolecular tracer.

To further examine whether the hyperpermeability of xenograft vessels is affected by the necessary skin incision and may thus relate to response to injury, we applied the same protocol as described for muscles by inducing and monitoring xenografts in both hind limbs. Images taken from the second tumor site (Fig. 3c, $13.5 \mathrm{~min}$ after tracer injection) document that tracer extravasation already occurred prior to the incision and first measurement at this location. The extravascular space shows a high fluorescence intensity which does not further increase as a function of time implicating the absence of an acute reaction. The resulting low-contrast images were not sufficient to segment tumor vessels and determine $I_{\mathrm{V}}$. Accordingly, exact $I_{\mathrm{En}}$ and leak index values could not be determined. However, to gain an impression, we used the mean values of vessel fraction $(1-p)$ and $I_{\mathrm{V}}$ of xenografts from the first site to estimate $I_{\mathrm{B}}$ and $I_{\mathrm{En}}$ for the second site. In conclusion, extravasation of the fluorescent tracer had started directly after injection and was independent of the time point of wounding, reflecting the chronic hyperpermeable phenotype $(\mathrm{CVH})$ of tumor vasculature.

\section{Vessel Morphology and Macromolecule Extravasation Throughout Xenograft Growth}

We repeatedly monitored vasculature and permeation as therapeutically relevant parameters in HCT-116 xenografts during growth at three different size ranges. With respect to tracer extravasation, tumors exhibited higher leak indices at smaller sizes (I and II) compared to the large volume (size III, Fig. 4a). A significant negative correlation between leak index and tumor volume was observed (Fig. 4b). Nevertheless, after reaching size III all xenografts still showed extravasation of the $150 \mathrm{kDa}$ FITC-dextran as is reflected by mean leak index values which were significantly higher compared to non-leaky vessels in normal tongue (Fig. 4a). This indicates that vascular hyperpermeability was still present.

Vascular fraction did not markedly differ between the three tumor sizes (Table 1). However, dilated vessels with diameters from 50 to $100 \mu \mathrm{m}$ were found in tumors of all sizes but with higher frequency in larger xenografts. Excessively enlarged vessels with diameters over $100 \mu \mathrm{m}$ were not present in small tumors but common when xenografts reached size III (Table 1). Tumors without dilated and ultradilated vessels showed significantly higher leak index values (21.5 min after tracer injection) compared to those with dilated vessels $(3.95 \pm 2.0$ and $1.17 \pm 0.7$, respectively, $p<0.05$, see also Suppl. Fig. S6). The data indicate that xenograft size, incidence of dilated vessels, and tracer permeation are related in the HCT-116 model.

To verify these findings, we studied a second subcutaneous xenograft model (SW620) with pCLE based on our protocol. Again, measurements were performed longitudinally at three size ranges. The vascular fraction in size I SW620 xenografts was slightly lower than in subcutaneous HCT-116 tumors. However, again no change in vascular fraction throughout tumor growth was observed (Table 1). Analogous to the HCT-116 model, SW620 tumors showed tracer extravasation at every size range. Again, we observed lower tracer leak indices when xenografts were larger (size II and III) as compared to the first measurement at size I (Fig. 5a) and found a correlation between tracer leakage and tumor volume in this xenograft model (Fig. 5b). The direct comparison of the two xenograft models reveals significant variances in tracer extravasation as is particularly seen in size II xenografts $\left(170-265 \mathrm{~mm}^{3}\right)$ with a mean leak index differing by a factor of 2 (21.5 min after tracer injection, $p<0.05$ ). Indeed, a significant decrease in tracer extravasation is seen in the SW620 tumors only between sizes I and II whereas the most prominent change in the HCT-116 model occurs between size II and III xenografts.

Similar to the HCT-116 model, small SW620 xenografts showed a lower frequency of dilated and ultradilated vessels than after reaching size II and III, although there was almost no difference between these larger sizes (Table 1). However, not all size III SW620 xenografts had dilated vessels in the examined FOVs and the incidence of ultradilated vessels was under $40 \%$ for both size II and size III tumors, as compared to $62.5 \%$ and $87.5 \%$ of the respective HCT-116 xenografts. Accordingly, tumor size correlated with both presence of dilated/ultradilated vessels and tracer leakage in the SW620 model, but no direct relationship between the two latter parameters was observed (Suppl. Fig. S6).

We further evaluated if the time interval between tumor cell injection and xenograft formation affects tracer extravasation. For this purpose, cell numbers ranging from 


\section{a Xenograft tumors - HCT-116}
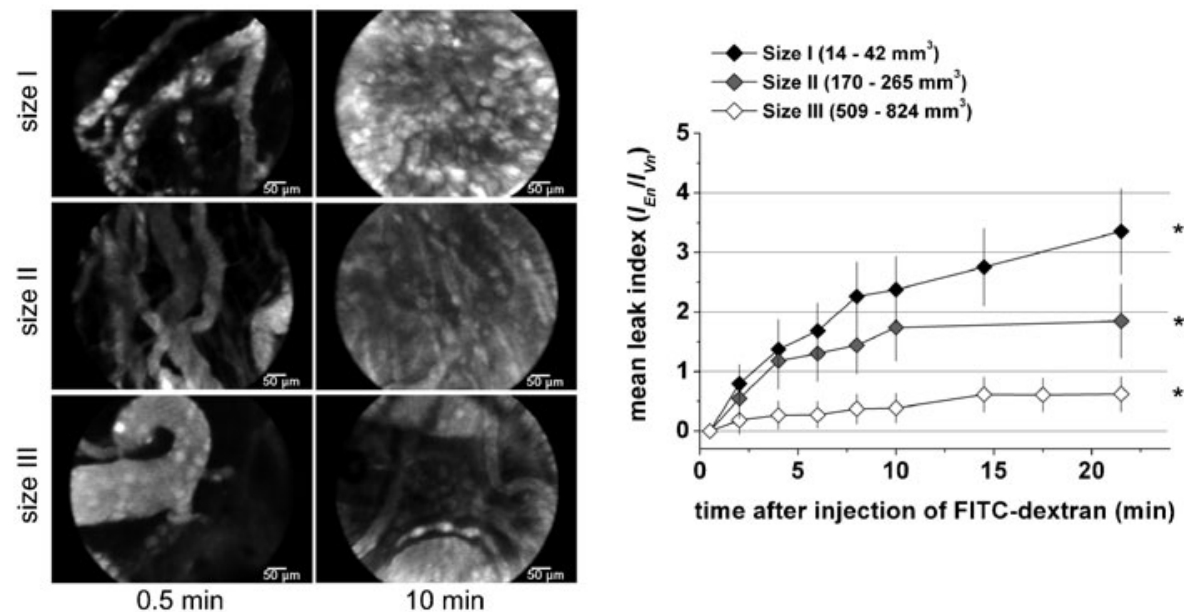

time after injection of FITC-dextran (min)

b

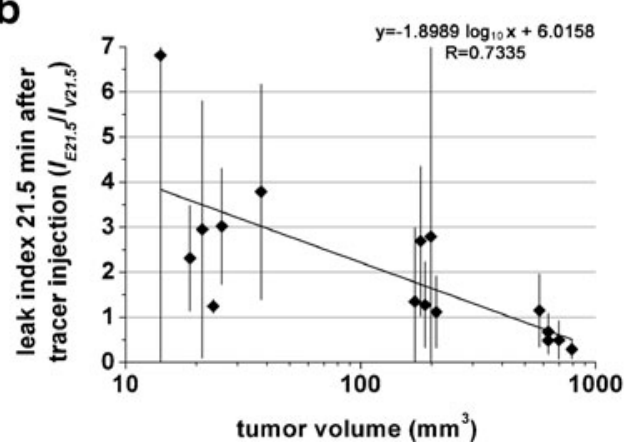

Fig. 4. HCT-116 xenografts show changes in extravasation of $150 \mathrm{kDa}$ FITC-dextran throughout subcutaneous growth in NMRI-nu/nu mice. a Representative images of vasculature of subcutaneous HCT-116 xenografts measured longitudinally during growth at three different sizes. Twelve tumors were included in the experiment. Images show the same site of injection 0.5 and $10 \mathrm{~min}$ after tracer injection. The graph documents the kinetics of the mean leak index $\left(I_{\mathrm{En}} / \mathrm{IVn}_{\mathrm{Vn}}\right)$ with error bars showing SDs from $n=8$ (size I) and $n=5$ (size II and III) tumors; leak indices significantly differ from normal tissue without tracer extravasation (see Fig. 1c; ${ }^{*} p<0.01$, two-sided Student's $t$ test). b The leak index values (21.5 min after tracer injection) correlate negatively with the tumor volume $(p<0.01$ for $f=n-2)$. Error bars show the intratumoral SD of each measurement.

500 to $1 \times 10^{5}$ SW620 cells were injected per site, and all xenografts were monitored when they had reached size I, i.e., between days 8 and 31. As documented in Fig. 5c, the time to develop a xenograft did not alter the vascular leak index. The reduction in tracer extravasation throughout xenograft growth as well as the increase in dilated and ultradilated vessels could thus be clearly attributed to tumor size and may be a common phenomenon in subcutaneous xenografts despite some cell and model type specific modifications.

Table 1. Mean proportion of vascular fraction $( \pm \mathrm{SD})$ in images taken from xenografts of different sizes 0.5 min after tracer injection and percentage of tumors with dilated and ultradilated vessels

\begin{tabular}{|c|c|c|c|c|c|c|c|}
\hline & & \multicolumn{3}{|l|}{ НCT-116 } & \multicolumn{3}{|l|}{ SW620 } \\
\hline & & Size $\mathrm{I}^{\mathrm{a}}$ & Size II $^{\mathrm{b}}$ & Size III $^{\mathrm{c}}$ & Size $\mathrm{I}^{\mathrm{a}}$ & Size II $^{\mathrm{b}}$ & Size III $^{\mathrm{c}}$ \\
\hline \multirow{5}{*}{ Tumors with } & Vascular & $33.7 \pm 4.6 \%$ & $\begin{array}{l}38.5 \pm 3.5 \% \\
(n=5)\end{array}$ & $36.0 \pm 5.2 \%$ & $31.3 \pm 3.9 \%$ & $30.6 \pm 5.5 \%$ & $31.6 \pm 4.3 \%$ \\
\hline & Dilated vessels & $62.5 \%$ & $87.5 \%$ & $100 \%$ & $\begin{array}{l}(n-15) \\
60 \%\end{array}$ & $81.8 \%$ & $87.5 \%$ \\
\hline & $(50-100 \mu \mathrm{m})$ & $(5 / 8)$ & $(7 / 8)$ & $(8 / 8)$ & $(9 / 15)$ & $(9 / 11)$ & $(7 / 8)$ \\
\hline & Ultradilated vessels & $0 \%$ & $62.5 \%$ & $87.5 \%$ & $13.3 \%$ & $36.4 \%$ & $37.5 \%$ \\
\hline & $(>100 \mu \mathrm{m})$ & $(0 / 8)$ & $(5 / 8)$ & $(7 / 8)$ & $(2 / 15)$ & $(4 / 11)$ & $(3 / 8)$ \\
\hline
\end{tabular}

At least seven different FOVs were included in the analysis for each tumor and size

${ }^{\mathrm{a}} 14-42 \mathrm{~mm}^{3}$

${ }^{\mathrm{b}} 170-265 \mathrm{~mm}^{3}$

${ }^{\mathrm{c}} 509-824 \mathrm{~mm}^{3}$ 

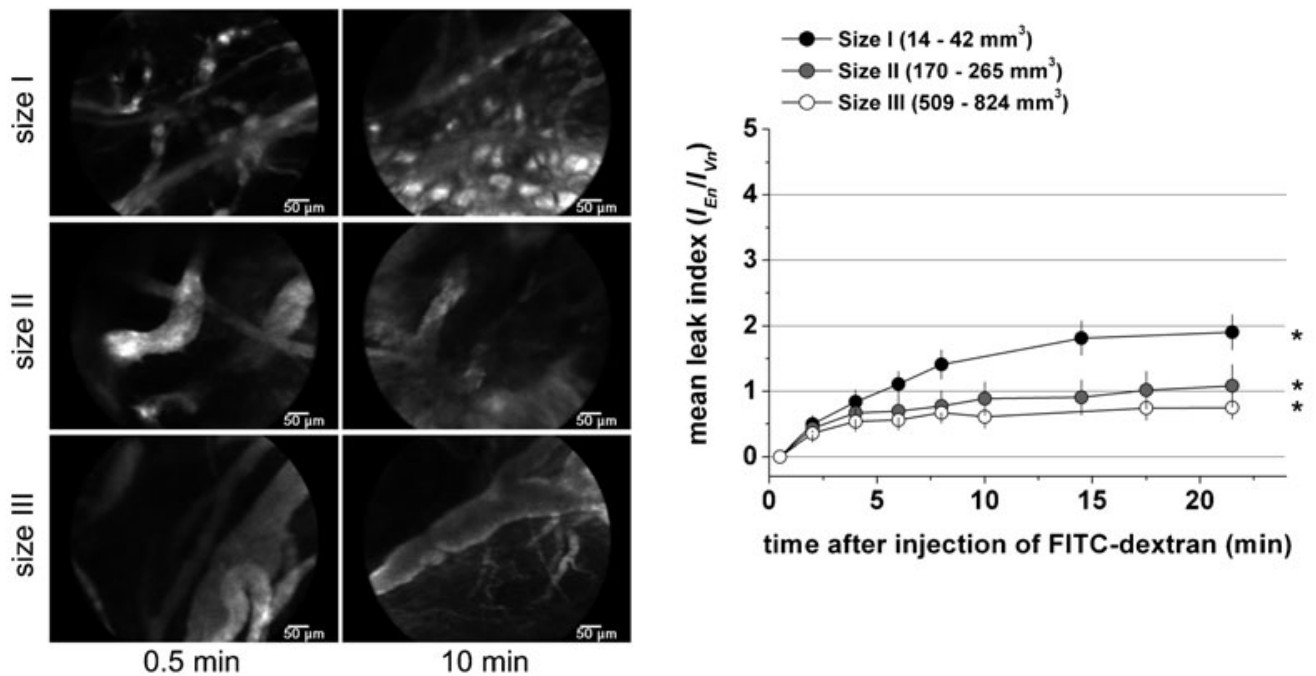

b

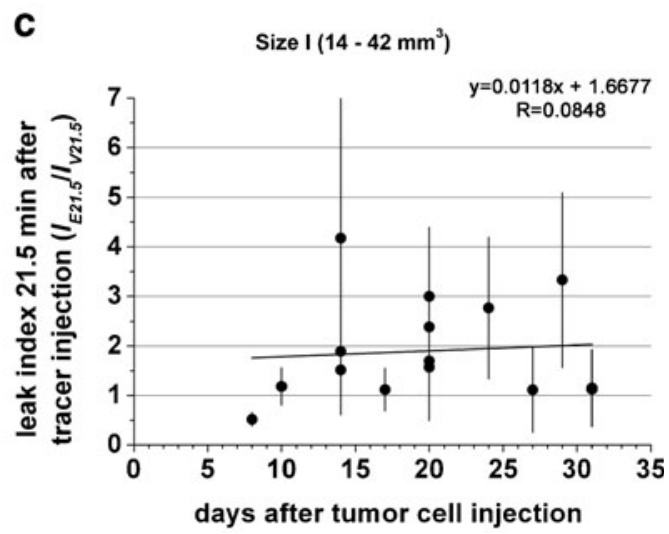

Fig. 5. Tracer extravasation in SW620 xenografts correlates with tumor volume but is independent of developmental period. a Representative images of vasculature of subcutaneous SW620 xenografts measured longitudinally during growth at three different sizes. Twenty-three tumors were included in the experiment. Images show the same site 0.5 and 10 min after tracer injection. The graph documents the kinetics of the mean leak index $\left(I_{\mathrm{En}} / I_{\mathrm{Vn}}\right)$ with error bars showing SDs from $n=15$ (size $\left.\mathrm{I}\right), n=$ 11 (size II), and $n=8$ (size III) tumors. Leak indices significantly differ from normal tissue without tracer extravasation $\left({ }^{*} p<0.01\right.$, two-sided Student's $t$ test). b The leak index ( $21.5 \mathrm{~min}$ after tracer injection) correlate negatively with the tumor volume $(p<0.01$ for $f=n-2)$. c Leak index in size I SW620 xenografts (21.5 min after tracer injection) as a function of time after tumor cell injection. Error bars in $\mathbf{b}$ and $\mathbf{c}$ show the intratumoral SD of each measurement.

\section{Discussion and Conclusion}

pCLE permits real-time confocal recording with high resolution and is only minimally invasive because of the small diameters of the microprobes [16]. Thus, it is applicable on standard disease models as shown in this study. This is the first demonstration of a consecutive application of pCLE throughout xenograft growth which remarkably reduced the number of animals.

The technology applied herein is suitable to monitor single vessels, even though it was not possible to find the exact same FOV at different time points during measurement. Anyway, due to the observed heterogeneity in the studied xenograft models the analysis of only one small area in few tumors would not reflect the characteristics of the vasculature. This is a common limitation of microscopic methods, especially if ROIs are manually positioned in every investigated frame [18]. Therefore, we aimed to average data from different FOVs for each tissue by using a large ROI and mean values for intravascular signal and vessel fraction to estimate the average extravascular fluorescence intensity. This could be realized by moving the small-sized microprobe over the tissue surface, thereby imaging up to 20 different FOVs (= 100 analyzed frames) per tumor and time point. A vessel segmentation algorithm was developed for image analysis and the leak index of $150 \mathrm{kDa}$ FITC-dextran was determined at defined time points after injection as an estimation of tracer permeation. This may not reflect vessel permeability alone, as other parameters also contribute to the amount of extravasated and accumulated 
macromolecules. However, it gives an estimate of tracer delivery to different tissues.

The presented protocol for pCLE could thus be applied to monitor therapy responses as a function of drug delivery but may also support studies on therapeutic strategies directed towards tumor vasculature in vivo. Moreover, a multitude of therapeutic antibodies are becoming promising tools in cancer treatment, and it is possible to use fluorochromelabeled variants in vivo during pCLE [21] and thereby pCLE could provide information on their delivery to the target tissue throughout therapy testing. It will also be useful to investigate other pathophysiological aspects, e.g., the role of the tumor micromilieu on the vasculature. There is accumulating evidence, for example, that tumor-associated fibroblasts contribute to the angiogenic switch in tumors [22], but their genuine role in vivo remains unclear. We previously described an accelerating effect of fibroblasts on tumor formation when co-injected with HCT-116 cells into NMRI$\mathrm{nu} / \mathrm{nu}$ mice [23]. The developed protocols for pCLE now offer the possibility to monitor vascular networks in such co-injection xenograft models.

In the present study, we examined the extravasation of $150 \mathrm{kDa}$ FITC-dextran of normal and tumor vasculature in mice as a model for macromolecular tracer permeation. Healthy microvessels of tongues under normal physiological conditions were impermeable to the tracer as expected for normal vessels possessing only BVP. This reveals that no spurious effects are triggered by the measuring procedure itself, e.g., vasodilation caused by phototoxicity or anaphylactic reactions which were reported for higher doses of injected FITC-dextran [24]. Upon induction, i.e., pinching of the tongue or skin incision at the femur muscle site, a strong tracer leakage was observed. In both cases, hyperpermeability was acute (AVH) because it was terminated within $60 \mathrm{~min}$ in tongues and depended on time of injury in muscles. Here, the skin incision provoked a massive influx of FITC-dextran leading to mean leak index values which were 9-fold higher compared to HCT-116 xenograft tissue. Accordingly, hyperpermeability of tumor vessels in the xenograft model was a chronic process $(\mathrm{CVH})$ since it was independent of the time of skin incision. This phenomenon, which was reported for many tumors [25, 26], is presumably caused by the presence of proangiogenic and permeabilizing factors triggering the EPR effect [8]. High plasma levels of VEGF have for example been shown in HCT-116 xenograft-bearing nude mice [27]. This may be accompanied by basement membrane degradation, pericyte detachment, and extensive vessel enlargement leading to tortuous architecture of the vessel network and vascular hyperpermeability $[1,28,29]$.

We observed a high intra- and intertumoral heterogeneity of the vascular morphology and grade of tracer permeation in xenografts of both colorectal cell line models. This may reflect diverse and heterogenic mechanisms of new vessel formation which depend on unbalanced secretion of angiogenic factors $[3,29,30]$. Interestingly, an increase in the proportion of dilated vessels throughout tumor growth was recognized. These are hypothesized to reflect so-called mother vessels - less mature vessels with thin walls and low pericyte coverage [3, 28, 29]. Extravasation of the $150 \mathrm{kDa}$ FITC-dextran, however, was found to decrease during xenograft growth which may demonstrate vascular maturation or the development of vascular malformations with muscular coat [3], both contradicting the mother vessel theory. Furthermore, the incidence of dilated/ultradilated vessels correlated with lower leak indices in the HCT-116 but not the SW620 model indicating that diverse mechanisms are responsible for permeation changes during xenograft growth.

The accumulation of macromolecules in tumors not only depends on vascular permeability but is also affected by interstitial fluid pressure (IFP) and lymphatic abnormalities $[12,30]$. It seems reasonable that the higher fluorescence signal in smaller xenografts results from a strong accumulation of fluorescent dye in the interstitium which is prevented by a high IFP in larger tumors. Indeed, it was claimed that high IFP values are present in solid tumors [31] and may lead to decreased uptake of plasma molecules and high molecular weight drugs [32]. Therefore, the decreased leak index values throughout tumor growth might not be due to matured vessel characteristics but could instead be caused by an increasing IFP despite vascular hyperpermeability. Some previous reports imply that IFP increases with tumor size [33], but others raise doubts that this is a general feature [34] as the relationship of IFP and angiogenic abnormalities was found to be very complex and depended on the tumor model [14].

Our results on vascular morphology and extravasation suggest that the selection of the xenograft model and its size may among others be critical for drug delivery and thereby affects outcome of therapy testing. Although there are diverse explanations for tumor size-dependent alterations in tracer leakage, the phenomenon itself may be common in experimental tumors and has to be taken into account. It is commonly agreed that orthotopic models are superior to subcutaneous xenografts, but an obstacle is their restricted accessibility for high-resolution imaging. The pCLE microprobes are flexible and can thus also be used endoscopically, so that some orthotopic models would become accessible for (vessel) monitoring $[35,36]$. The presented procedure can be adapted to the respective requirements, e.g., we already realized to monitor the vasculature in an orthotopically grown rectal xenograft in a preliminary experiment (data not shown). However, extended studies will be required to verify if orthotopic tumors also show size-related modulations of vascular structures and extravasation.

Acknowledgments. This work was supported by the German Research Foundation (INST 269/319-1 FUGG, SCHN 430/3-3 and SCH 430/6-1), the Saxon State Ministry of Science and the Fine Arts (PhD fellowship and institutional), and the National Cancer Institute of Canada-Terry Fox Foundation. OncoRay is funded by the BMBF in the program "Center for Innovation Competence". 
Conflict of Interest Statement. The authors declare that they have no conflict of interest.

\section{References}

1. Carmeliet P, Jain RK (2000) Angiogenesis in cancer and other diseases. Nature 407:249-257

2. Nagy JA, Benjamin L, Zeng H, Dvorak AM, Dvorak HF (2008) Vascular permeability, vascular hyperpermeability and angiogenesis. Angiogenesis 11:109-119

3. Nagy JA, Chang SH, Dvorak AM, Dvorak HF (2009) Why are tumour blood vessels abnormal and why is it important to know? Br J Cancer $100: 865-869$

4. Dvorak HF (1986) Tumors: wounds that do not heal. Similarities between tumor stroma generation and wound healing. N Engl J Med 315:1650-1659

5. Dalgleish AG, O'Byrne K (2006) Inflammation and cancer: the role of the immune response and angiogenesis. Cancer Treat Res 130:1-38

6. Dvorak AM, Feng D (2001) The vesiculo-vacuolar organelle (VVO). A new endothelial cell permeability organelle. J Histochem Cytochem 49:419-432

7. Noguchi Y, Wu J, Duncan R et al (1998) Early phase tumor accumulation of macromolecules: a great difference in clearance rate between tumor and normal tissues. Jpn J Cancer Res 89:307-314

8. Fang J, Nakamura H, Maeda H (2011) The EPR effect: unique features of tumor blood vessels for drug delivery, factors involved, and limitations and augmentation of the effect. Adv Drug Deliv Rev 63:136-151

9. Vajkoczy P, Ullrich A, Menger MD (2000) Intravital fluorescence videomicroscopy to study tumor angiogenesis and microcirculation. Neoplasia 2:53-61

10. McDonald DM, Choyke PL (2003) Imaging of angiogenesis: from microscope to clinic. Nat Med 9:713-725

11. Fukumura D, Jain RK (2008) Imaging angiogenesis and the microenvironment. APMIS 116:695-715

12. Dreher MR, Liu W, Michelich CR et al (2006) Tumor vascular permeability, accumulation, and penetration of macromolecular drug carriers. J Natl Cancer Inst 98:335-344

13. Hak S, Reitan NK, Haraldseth O, de Lange DC (2010) Intravital microscopy in window chambers: a unique tool to study tumor angiogenesis and delivery of nanoparticles. Angiogenesis 13:113-130

14. Simonsen TG, Gaustad JV, Leinaas MN, Rofstad EK (2012) High interstitial fluid pressure is associated with tumor-line specific vascular abnormalities in human melanoma xenografts. PLoS One 7:e40006

15. Staton CA, Reed MW, Brown NJ (2009) A critical analysis of current in vitro and in vivo angiogenesis assays. Int J Exp Pathol 90:195-221

16. Laemmel E, Genet M, Le Goualher G et al (2004) Fibered confocal fluorescence microscopy (Cell-viZio) facilitates extended imaging in the field of microcirculation. A comparison with intravital microscopy. J Vasc Res 41:400-411

17. Lin KY, Maricevich M, Bardeesy N, Weissleder R, Mahmood U (2008) In vivo quantitative microvasculature phenotype imaging of healthy and malignant tissues using a fiber-optic confocal laser microprobe. Transl Oncol 1:84-94

18. Faye N, Fournier L, Balvay D et al (2011) Dynamic contrast enhanced optical imaging of capillary leakage. Technol Cancer Res Treat 10:49-57
19. Kelland LR (2004) Of mice and men: values and liabilities of the athymic nude mouse model in anticancer drug development. Eur J Cancer 40:827-836

20. Peters T, Sindrilaru A, Hinz B et al (2005) Wound-healing defect of CD18(-/-) mice due to a decrease in TGF-betal and myofibroblast differentiation. EMBO J 24:3400-3410

21. Nakai Y, Shinoura S, Ahluwalia A, Tarnawski AS, Chang KJ (2012) Molecular imaging of epidermal growth factor-receptor and survivin in vivo in porcine esophageal and gastric mucosae using probe-based confocal laser-induced endomicroscopy: proof of concept. J Physiol Pharmacol 63:303-307

22. Marsh T, Pietras K, McAllister SS (2012) Fibroblasts as architects of cancer pathogenesis. Biochim Biophys Acta. doi:10.1016/ j.bbadis.2012.10.013

23. Schmid SA, Dietrich A, Schulte S, Gaumann A, Kunz-Schughart LA (2009) Fibroblastic reaction and vascular maturation in human colon cancers. Int J Radiat Biol 85:1013-1025

24. Richter W (1969) Increased vascular permeability in mice induced by dextran. A comparison with the anaphylactoid reaction in rats. Acta Pharmacol Toxicol (Copenh) 27:331-348

25. Dvorak HF, Nagy JA, Dvorak JT, Dvorak AM (1988) Identification and characterization of the blood vessels of solid tumors that are leaky to circulating macromolecules. Am J Pathol 133:95-109

26. Yuan F, Dellian M, Fukumura D et al (1995) Vascular permeability in a human tumor xenograft: molecular size dependence and cutoff size. Cancer Res 55:3752-3756

27. Keyes KA, Mann L, Cox K et al (2003) Circulating angiogenic growth factor levels in mice bearing human tumors using Luminex Multiplex technology. Cancer Chemother Pharmacol 51:321-327

28. Pettersson A, Nagy JA, Brown LF et al (2000) Heterogeneity of the angiogenic response induced in different normal adult tissues by vascular permeability factor/vascular endothelial growth factor. Lab Invest 80:99-115

29. Dvorak HF (2002) Vascular permeability factor/vascular endothelial growth factor: a critical cytokine in tumor angiogenesis and a potential target for diagnosis and therapy. J Clin Oncol 20:4368-4380

30. Fukumura D, Jain RK (2007) Tumor microenvironment abnormalities: causes, consequences, and strategies to normalize. J Cell Biochem 101:937-949

31. Heldin CH, Rubin K, Pietras K, Ostman A (2004) High interstitial fluid pressure - an obstacle in cancer therapy. Nat Rev Cancer 4:806-813

32. Jain RK (2001) Delivery of molecular and cellular medicine to solid tumors. Adv Drug Deliv Rev 46:149-168

33. Gutmann R, Leunig M, Feyh J et al (1992) Interstitial hypertension in head and neck tumors in patients: correlation with tumor size. Cancer Res 52:1993-1995

34. Lunt SJ, Kalliomaki TM, Brown A et al (2008) Interstitial fluid pressure, vascularity and metastasis in ectopic, orthotopic and spontaneous tumours. BMC Cancer 8:2

35. Ignat M, Aprahamian M, Lindner V et al (2009) Feasibility and reliability of pancreatic cancer staging using fiberoptic confocal fluorescence microscopy in rats. Gastroenterology 137(1584-1592): e1581

36. von Delius S, Feussner H, Wilhelm D et al (2007) Transgastric in vivo histology in the peritoneal cavity using miniprobe-based confocal fluorescence microscopy in an acute porcine model. Endoscopy 39:407-411 